\title{
Les réseaux sociaux numériques pour chercheurs : quelles pratiques, quels enjeux?
}

Cécile Arènes

Conservateur des bibliothèques

Les réseaux sociaux numériques pour chercheurs connaissent, depuis 2010, une croissance exponentielle. Les chercheurs s'y inscrivent pour plusieurs raisons : ces outils leur fournissent une page clé en main, qui résume leur parcours et présente leur bibliographie. Ils sont nombreux à juger ces sites utiles pour des coopérations internationales. Leur utilisation massive soulève toutefois plusieurs problèmes d'ordre éthique. Le devenir des documents déposés sur ces réseaux est incertain : les conditions d'utilisation de certains sites sont prédatrices en matière de données personnelles. Pour les institutions, le recours massif à ces réseaux par les chercheurs n'est pas anodin : elles courent le risque de voir capté sur des plateformes qu'elles ne maîtrisent pas le produit de la recherche qu'elles ont financé.

Since 2010, social networking sites for academics and researchers have shown an exponential growth. Researchers sign up for many different reasons : these tools provide them with a customizable profile page, which outlines their career and displays their list of publications. Many find these sites useful for international collaboration. However, this kind of widespread use raises many ethical issues. What becomes of uploaded documents remains uncertain, as the terms of use of some sites allow predatory reuse of personal data. For research institutions, the unrestricted use of social networks by their researchers is not insignificant, as the risk exists that the research output that they financially support will be appropriated by online platforms that they can't control.

Réseaux sociaux

Communication

Open access

Visibilité

Identité numérique

Social network

Communication

Open access

Visibility

Online identity 
Les réseaux sociaux numériques de la recherche connaissent un succès qui ne se dément pas depuis quelques années. Dans la continuité des réseaux généralistes, ils permettent aux chercheurs du monde entier d'être connectés et d'échanger. A l'heure actuelle, ce sont près de $68 \%$ des Français qui sont inscrits sur un réseau social et ils y consacrent près d'une heure trente par jour (1). Le phénomène, qui va en s'accentuant, n'est pas à négliger. En effet, beaucoup d'utilisateurs relaient par ce biais des informations variées, à la fois insolites et banales, mais parfois aussi problématiques ou essentielles dans le cadre professionnel. Selon Danah Boyd, spécialiste des médias sociaux et chercheur au Data \& Society et à Microsoft Research, les réseaux sociaux numériques sont principalement des outils destinés à créer du lien : « Un site de réseau social est une catégorie de site web avec des profils d'utilisateurs, des commentaires publics semi-persistants sur chaque profil, et un réseau social public navigable (traversable) affiché en lien direct avec chaque profil individuel » (2). Le succès de tels sites réside sans aucun doute dans la facilité qu'ont les membres à gagner des contacts et à échanger presque instantanément.

Qu'il s'agisse d'échanger autour d'une profession, d'intérêts communs, les réseaux sociaux sont souvent jugés comme des outils qui aident aux rapprochements entre les individus, mais aussi comme des plateformes prédatrices des données de leurs utilisateurs, au mépris de tout respect de la vie privée.

Dans le cadre de l'activité scientifique, tout l'enjeu autour de ces outils réside dans leur extrême simplicité d'utilisation, par les incitations permanentes à y publier, alors que le devenir des données déposées par les utilisateurs n’a jamais été plus incertain. Plusieurs questions se posent à leur sujet : d'une part, ces sites constituent des silos fermés qui captent les informations; ils ne sont pas interopérables, ce qui cause un vrai problème pour la diffusion scientifique. D’autre part, le dépôt massif de publications sur les réseaux sociaux soulève des interrogations d'ordre divers, telles que les données personnelles, les droits liés aux publications scientifiques, le positionnement des institutions face à ces nouveaux acteurs. Dans le même temps, le succès que connaissent ces plateformes auprès 
des chercheurs révèle un besoin de visibilité au niveau international, que les outils traditionnels ne peuvent pas leur apporter à l'heure actuelle.

\section{La présence numérique des chercheurs}

Le travail scientifique s'est toujours articulé autour d'échanges nourris entre les chercheurs, qui ont eu recours au travail en réseau de longue date. Pour autant, au tournant des années 2010 sont apparues des logiques de présence en ligne de plus en plus individuelles.

\section{Quelles traces sur la Toile?}

On parle souvent d'identité numérique pour désigner les signes d'un individu sur la toile. A ce terme, Louise Merzeau, maître de conférences en sciences de l'information à Paris Ouest, préfère celui de « présence » (3). Elle le juge préférable dans la mesure où l'activité sur la toile d'un individu, qui est la corrélation entre les traces qu'il y laisse et les identifiants qui lui sont propres, est souvent loin de dessiner une personnalité complète. L'identité numérique peut se révéler parcellaire ou tronquée. De fait, s'en tenir à la présence numérique apparaît plus pertinent. Pour Louise Merzeau, la présence numérique des chercheurs se caractérise désormais par un étoilement. On ne peut trouver une page, un faisceau de traces central, d'où découleraient tous les autres. Au contraire, c'est une présence en toile, ramifiée, qu'on observe, où certains espaces sont choisis pour constituer des carrefours. Cela requiert une certaine expertise de la part des scientifiques, qui doivent administrer leurs différents comptes et les relier entre eux. Une présence numérique aujourd'hui, a fortiori celle d'un chercheur, est caractérisée par des facettes nombreuses. II n'est pas rare, en effet, de recenser pour une même personne des publications sur des revues en ligne et en archives ouvertes, un blog, un ou plusieurs réseaux sociaux, généralistes et scientifiques, un logiciel de références bibliographiques, parfois un site personnel. Chaque chercheur choisit, dans une logique de complémentarité, les différents espaces les plus adaptés, tant à ses besoins qu'au réseau de pairs qui s'y trouve déjà, pour s'assurer visibilité et contacts (4). Eric Verdeil, géographe, chercheur au CNRS, et inscrit sur plusieurs de ces plateformes, relève que la mise à jour et les échanges sur les réseaux sociaux est de moins en moins 
une activité secondaire, mais qu'elle fait au contraire partie intégrante de l'activité professionnelle des chercheurs (5). Lorsqu'on se penche sur les pratiques des chercheurs, on s'aperçoit en effet que I'utilisation de ces sites est régulière, de l'ordre de trois à quatre consultations par mois (6). II reste que la gestion de ces outils, toujours plus nombreux, est chronophage et demande des compétences en matière de communication que peu de chercheurs ont acquises dans leurs cursus académiques, dont les enseignements sont encore très marqués par le modèle traditionnel des publications scientifiques.

\section{Internet, un réseau créé par le scientifiques, adapté à leurs besoins}

Le modèle de l'article publié dans une revue académique n'a que peu évolué depuis le Journal des Sçavans, puis du modèle de Henry Oldenburg à la Royal Society ${ }^{1}$. Sa fonction principale, consigner par écrit une découverte ou une idée et ainsi la protéger, avant de la partager, crée les conditions de naissance d'une communauté de pairs dont les échanges sont aussi nombreux que fréquents. Le travail en réseau de la communauté scientifique n'est pas né à la fin du $20^{\mathrm{e}}$ siècle et il a toujours su profiter des techniques de son époque pour s'exercer pleinement. Pour autant, l'évolution des technologies de la communication, et notamment l'arrivée de l'Internet, ont bouleversé la donne. L’Internet a été pensé par des scientifiques afin de répondre à leurs propres besoins. Les premières expériences sur un réseau d'échange entre les ordinateurs datent des années 1960 et sont le produit conjoint des besoins des militaires et des recherches scientifiques. Les universitaires se sont très vite penchés sur la question de l'échange des idées. Rapidement, les premiers espaces de discussion en ligne sont apparus et les premiers forums étaient constitués d'universitaires du monde entier, venus échanger et débattre. Ils ont constitué pour la communauté scientifique une sorte d’idéal, ainsi que le rappelle Patrice Flichy, professeur de sociologie à l'université Paris Est, dans la mesure où les scientifiques ont conçu pour eux-mêmes des espaces adaptés. Usenet, pionnier des systèmes de

\footnotetext{
${ }^{1}$ Fondé en 1665 , le Journal des Sçavans est considéré comme le premier périodique scientifique en Europe. C'est sur ce modèle qu'Henry Oldenburg fonde quelques mois plus tard Philosophical Transcations of the Royal Society. Ces deux titres vont contribuer à la diffusion de la connaissance scientifique. Le Journal des Sçavans est consultable sur la bibliothèque numérique de la BnF, Gallica : http://catalogue.bnf.fr/ark:/12148/cb343488023
} 
forums en réseau, « est non seulement le média de l'échange intellectuel mais également le résultat d'une coopération technique permanente » (7).

Rappelons aussi, que Facebook, le premier réseau social numérique connu du grand public, apparu en 2004, est né dans une université, créé par un étudiant pour ses camarades. Réservé durant quelques années aux étudiants d'Harvard, qui s'en servaient essentiellement pour se retrouver sur le campus, le site s'est ensuite ouvert progressivement sur invitation, avant d'accepter toutes les inscriptions. Le monde universitaire a, de fait, toujours été très lié à l'émergence des réseaux sociaux et les plateformes d'aujourd'hui ne sont finalement que la continuation de ces espaces d'échanges, avec une nouveauté de taille, la mise en avant des personnes.

\section{Quelles motivations pour s'inscrire aujourd'hui?}

Les motivations pour s'inscrire sur des plateformes d'échanges se sont profondément transformées. Désormais, la mise en avant de l'individu prend le pas sur le travail du laboratoire. Pour Julien Pierre, l'identité numérique personnelle, que les chercheurs valorisent sur les réseaux sociaux numériques de la recherche, a deux raisons principales. En premier lieu, la compétitivité accrue du monde de la recherche ces dernières années a rendu nécessaire le fait d'être visible. En second lieu, nombreux sont les chercheurs qui sont avides d'espaces d'écriture qu'ils maîtrisent et où ils se sentent plus libres (8). De ce point de vue, les réseaux sociaux, qui permettent de se créer en très peu de temps une sorte de carte de visite augmentée, apparaissent aux yeux des chercheurs, et notamment des plus jeunes, comme des outils adaptés pour se faire connaître et pour rechercher un poste.

\section{Des premiers réseaux sociaux aux réseaux sociaux numériques de}

\section{la recherche}

Les chercheurs sont présents à la fois sur les réseaux sociaux généralistes et sur les réseaux sociaux spécialisés. Sur ces différents outils, ils n'ont pas les mêmes pratiques, ni les mêmes desseins, tout en 
étant à la recherche des fonctionnalités offertes par l'ensemble de ces sites, les contacts et le contenus. Les réseaux de contact, tout d'abord, mettent des individus en relation; on peut citer Facebook comme exemple le plus connu. Les inscrits peuvent poster des messages sur leur profil ou sur celui de leurs contacts, de façon à ce qu'ils soient lus par un ensemble de personnes. Contrairement aux mails ou aux forums, qui concernent une communauté restreinte, les réseaux sociaux de contenu donnent les moyens à tout un chacun de pouvoir communiquer à plus vaste échelle. C'est notamment ce qui a constitué leur succès. Ce sont aussi des réseaux de partage de documents, quelle que soit leur nature; Flickr ou Instagram, pour les photos, en font partie. Là encore, le but est de diffuser largement des documents. Chaque publication figure sur le profil de l'utilisateur et ses contacts pourront la relayer et la partager pour lui garantir une plus grande audience.

\section{Les réseaux sociaux à l'université aujourd'hui}

Observant le succès des plateformes pour le grand public, de nombreuses institutions ont souhaité ouvrir des réseaux sociaux réservés à la communauté de leur établissement. Malgré un fort investissement pour certains d'entre eux, ces initiatives ne se développent pas massivement. Les chercheurs leur préfèrent des réseaux au champ plus vaste, où retrouver leurs pairs à l'échelle internationale.

En France, les chercheurs sont toujours plus nombreux à utiliser les réseaux sociaux numériques, quels qu'ils soient. D'après une étude citée par Aline Bouchard, conservateur à I'URFIST de Paris ${ }^{2}$, ils étaient $42 \%$ en 2011 et $70 \%$ en 2013 (9). Ils sont probablement davantage encore aujourd'hui. Facebook, Linkedln et Twitter sont les plus utilisés par les chercheurs. Twitter est spontanément cité par les chercheurs lorsqu'il s'agit d'évoquer un réseau social généraliste dont ils se servent pour leur recherche. Pour Aurélien Berra, maître de conférences à Paris-Ouest, « le réseau social Twitter est extrêmement important, par exemple. Il contribue à la veille scientifique, tout comme les fils RSS, mais aussi à l'identité et à la dynamique de réseau de cette communauté internationale » (10). Lors

\footnotetext{
${ }^{2}$ URFIST : unité régionale de formation à l'information scientifique et technique.
} 
d'une table ronde organisée à l'occasion de l'Open access week à Lyon en 2014, Pablo Jensen, physicien et directeur de recherche au CNRS, expliquait qu'il ne lui serait pas impossible aujourd'hui de délaisser la lecture de Nature au profit du suivi d'une quinzaine de chercheurs sur Twitter, pour se tenir au courant de l'actualité et des évolutions dans son domaine de recherche (11). Les scientifiques sont nombreux à être présents sur les réseaux généralistes ${ }^{3}$ et certains chercheurs français, comme Sylvain Deville, chargé de recherche au CNRS, n'hésitent pas à plaider pour une plus forte présence de leurs pairs de l'Hexagone sur ces sites (12).

Pour Arnaud Saint-Martin et Jérôme Lamy, rédacteurs d'un carnet de recherche sur la plate-forme Hypothèses ${ }^{4}$, diffuser la recherche sur des sites grand public va jusqu'à relever de l'« impératif moral » : « nous considérons qu'ayant la chance de faire un travail payé par le contribuable, nous nous devons à une certaine forme de publicisation régulière qui ne soit pas cantonnées aux revues académiques. [...] Nous nous faisons un devoir (le mot n'est pas trop fort) de publier nos recherches régulièrement et sur un support gratuitement accessible parce qu'il $y$, sous-jacent, une absolue nécessité de rendre ce que la communauté civique nous a donné (nos salaires et le temps de nous consacré à des activités de recherche). » (13)

\section{L'explosion des réseaux sociaux numériques de la recherche}

Nés quelques années après les blogs et les carnets de recherche, les réseaux sociaux numériques de la recherche voient leurs inscrits augmenter, pour des raisons assez disparates selon les chercheurs. Ces outils présentent la particularité de combiner systématiquement ces deux fonctionnalités de contact et contenu. Leurs fonctionnalités sociales permettent aisément aux chercheurs de se constituer un réseau de contacts. Sur les plus utilisés, ResearchGate et Academia.edu, on trouve aussi lié au profil de l'utilisateur un certain nombre de ses publications, qu'il est invité, sinon incité, à ajouter. C'est cette porosité des fonctions qui séduit les scientifiques du monde entier. En 2014, le

\footnotetext{
${ }^{3}$ On peut citer le physicien Stephen Hawking sur twitter https://twitter.com/steven_hawking ou encore Richard Dawkins sur facebook https://www.facebook.com/RichardDawkinsFoundation.

${ }^{4}$ Créée en 2009, cette plate-forme de carnets scientifiques héberge aujourd'hui près de mille blogs. Elle est maintenue par le CLEO, le Centre pour l'édition électronique ouverte, UMS existant depuis 2007.
} 
Consortium Couperin, structure qui regroupe les établissements universitaires et de recherche pour négocier et garantir l'accès à la documentation électronique, a conduit une vaste étude sur I'utilisation que font les chercheurs des réseaux sociaux numériques spécialisés. L'étude a révélé que près de $60 \%$ d'entre eux les connaissent et que $42 \%$ en sont utilisateurs. Ceux qui n'utilisent pas ces plateformes expliquent ne pas voir leur intérêt et disposer de trop peu de temps pour s'y pencher. Certains de leurs arguments ne vont pas sans rejoindre les limites pointées par les utilisateurs euxmêmes. Près d'un chercheur sur deux a donc aujourd'hui recours à ces outils. Parmi les inscrits sur ces sites, $70 \%$ y déposent leurs publications de recherche, alors qu'ils sont $86 \%$ à tout ignorer des conditions générales d'utilisation de ces plateformes (6).

Ce sont ResearchGate et Academia.edu qui se partagent la majorité des utilisateurs, le premier ayant la préférence des sciences exactes et des sciences du vivant, le second des sciences humaines et sociales. Academia.edu, dont le siège est aux Etats-Unis, prospère sur une autorité mal acquise : le « .edu » de son adresse, acheté avant que cette extension ne soit réservée aux institutions, l'assimile souvent à des outils portés par les institutions, alors qu'il s'agit d'une entreprise privée, dont les levées de fonds n'ont rien à envier à celles de Twitter et Facebook. D'autres plateformes existent, comme MyScienceWork, entreprise dont le siège est au Luxembourg et dont les méthodes ont fait rapidement débat (14).

A ces réseaux scientifiques généralistes s'ajoutent des réseaux disciplinaires. On relève toutefois qu'un réseau pionnier comme BioMedExpert a fusionné avec Mendeley, le réseau de références bibliographiques appartenant aujourd'hui à l'éditeur scientifique Elsevier. Les initiatives disciplinaires sont réservées à des communautés plus restreintes et parfois regroupées autour d'une seule thématique, comme par exemple Malaria world qui fédère les chercheurs autour de cette maladie (15). 


\section{Quelles pratiques en sciences de la vie?}

L'étude conduite par Couperin révèle que les chercheurs en sciences de la vie sont moins inscrits que d'autres disciplines sur les réseaux sociaux généralistes. En revanche, ils sont en tête en ce qui concerne les réseaux sociaux numériques de la recherche, avec un taux d'inscriptions à $47 \%$ et une connaissance de ces outils à $67 \%$ et ils figurent parmi les utilisateurs de ces sites les plus satisfaits. Autre particularité de la discipline, les chercheurs ont recours moins aux réseaux sociaux numériques de la recherche pour une utilisation spécifique liée à leur domaine de recherche (40\%) que pour une utilisation générale (59\%). Les bibliothécaires auteurs de cette étude, enfin, n’ont pas manqué de relever qu'ils figuraient aussi parmi les moins gros déposants dans des archives ouvertes, alors même que ce dépôt devient une condition indispensable pour l'obtention d'un financement européen. II en résulte pour les auteurs un nécessaire accompagnement à conduire (6).

\section{Des atouts pour ces nouveaux outils?}

Les atouts des réseaux sociaux numériques de la recherche sont indéniables pour les chercheurs. Dans une étude réalisée sur ces plateformes, Emma Bester, doctorante au CNAM, rapporte que c'est le service de profil qui est recherché, suivi du service de plateforme qui permet de suivre l'activité des membres. Le fil d'actualités permet de découvrir les publications récentes de ses contacts. Les recommandations et les nombreuses notifications sont également prisées, dans la mesure où elles permettent de ne pas manquer de nouveautés. Les membres apprécient également de pouvoir classer leurs publications sur le profil en fonction de catégories assez fines, correspondant à leurs besoins (16). Les outils de profilage de ces réseaux permettent aux utilisateurs de recevoir de nombreuses suggestions sur d'éventuels contacts à ajouter. De même, leurs moteurs de recherche donnent la possibilité de rechercher des publications comme des personnes, dans une discipline donnée. Dernier service très apprécié, les statistiques qui permettent de connaître le nombre de vues et de téléchargements sur une publication, ainsi que les consultations du profil par les autres membres (6). 


\section{L'exemple de ResearchGate}

ResearchGate, le réseau social de chercheurs le plus utilisé en sciences exactes et en sciences de la vie, existe depuis 2008 et il affiche un million et demi de membres. Le site a été imaginé et créé par des chercheurs. ResearchGate dispose des fonctionnalités classiques des réseaux sociaux, auxquelles s'adjoignent quelques spécificités. Depuis 2012, la plateforme a mis en place un "RG score » pour ses utilisateurs. Il est entre autres calculé sur la base des interactions d'un membre avec ses contacts, du nombre de publications déposées, des questions auxquelles il répond. Le titre des revues dans lesquelles sont publiés les articles fait partie du calcul du « RG score ». Parmi les fonctions en cours de développement, la fonction "Open review ", qui existe depuis un an, donne la possibilité aux inscrits d'évaluer spontanément les documents de leurs contacts. De son côté, chaque membre peut lui-même demander une évaluation à son réseau, s'il le souhaite. Contrairement à d'autres réseaux pour chercheurs, il n'est pas possible de renseigner disciplines et compétences librement sur son profil. Cette contrainte aboutit toutefois à une relative harmonisation des pratiques et à une recherche par sujet un peu plus pertinente que dans d'autres réseaux spécialisés. Les membres du réseau peuvent recommander leurs contacts pour l'une ou l'autre de ses compétences. Les établissements sont eux aussi à sélectionner dans une liste, ce qui permet d'éviter les erreurs. En revanche, les laboratoires, eux, ne bénéficient pas d'une sélection préalable et il en résulte de nombreuses occurrences, plus ou moins erronées, pour une même structure, rendant leur recherche très malaisée. Par ailleurs, le site, qui affirme ne pas faire de profit avec les données déposées, fonctionne malheureusement en vase clos: les données déposées ne sont ni interopérables, ni réexploitables, de sorte qu'il est impossible de pousser plus avant des recherches. Difficile, par exemple, de retrouver dans ces conditions les publications d'un laboratoire donné sur ResearchGate et de les valoriser.

Forts d'une carte de visite clé en main, beaucoup de chercheurs estiment disposer malgré tout d'une sorte de vitrine de leurs activités. Ces outils leur offrent la possibilité de suivre des personnes connues dans leur discipline, ou avec qui ils aimeraient travailler (17). Ces sites sont aussi des recours 
pour les étudiants avancés qui travaillent dans des pays où les abonnements à la documentation électronique sont devenus trop onéreux (18). Toutes disciplines confondues, c'est la visibilité, suivie de la diffusion et du partage de contenus, ainsi que la recherche de collègues et de projets, qui sont considérés comme les principaux atouts de ces plateformes (6). Pour tous, la logique de création du profil relève de la mise en avant et elle est proche de la notion de personal branding ${ }^{5}$.

\section{La visibilité individuelle à quel prix?}

Les chercheurs eux-mêmes relèvent de nombreuses limites aux réseaux sociaux spécialisés qu'ils utilisent. L'éparpillement des sources est mentionné en premier lieu. On peut également citer le manque de fiabilité de ces plateformes, tant du point de vue de la protection des auteurs que de la pertinence des informations qui sont envoyées aux utilisateurs (6). Toutefois, si les inquiétudes concernant la fiabilité sont exprimées, elles ne modifient pas les pratiques des chercheurs, qui sont nombreux à déposer leurs travaux sur les réseaux sociaux.

\section{Données personnelles et confidentialité}

Les réseaux sociaux numériques de la recherche posent en effet le problème des données personnelles des chercheurs, qui les utilisent assidûment. Un document retiré du réseau Academia.edu, par exemple, n'apparaît plus sur le profil de l'utilisateur, mais il reste très probablement conservé par le site pour une utilisation incertaine. Si le siège de l'entreprise ResearchGate est en Allemagne, pays qui bénéficie de lois sur les données personnelles protectrices pour les utilisateurs, il n'en va pas de même pour son homologue nord-américain. Academia.edu, notamment, fait naître des inquiétudes quant à l'avenir des documents qui y sont téléchargés, les conditions générales d'utilisation du site ne laissant guère planer de doute : "By making any Member Content available through the Site or Services, you hereby grant to Academia.edu a worldwide, nonexclusive, transferable, sublicenseable, perpetual, royalty-free license to reproduce, modify for

\footnotetext{
${ }^{5}$ Anglicisme que l'on peut traduire par marque personnelle, le personal branding consiste à utiliser des techniques classiques de marketing et de communication pour les appliquer à une personne. Cette technique s'est développée avec l'apparition du web 2.0.
} 
formatting purposes, prepare derivative works based upon, publicly display, publicly perform, distribute, and otherwise use your Member Content in connection with operating and providing the Services and Content to you and to other Members" (19) ResearchGate, pour sa part, annonce une politique moins agressive et affiche des conditions d'utilisation plus avantageuses pour ses utilisateurs. Le site assure que son but n'est pas de faire des profits en utilisant leurs données et il garantit la confidentialité des éléments déposés. Toutefois, comme Academia.edu, il s'autorise le recours à des tiers, dont on ne sait pas s'ils sont soumis aux mêmes conditions. On connaît d'ailleurs le précédent de la vente du site de gestion bibliographique Mendeley, créé par des étudiants, à l'éditeur scientifique Elsevier, qui avait donné lieu à des protestations et des désinscriptions de la part de chercheurs inquiets du devenir de leurs données (20). De même, il n'est pas rare sur la Toile de voir des services fermer, laissant leurs utilisateurs sans aucun moyen d'accéder à leurs données et à leurs documents.

\section{Propriété des données produites}

Outre la question des données personnelles des chercheurs, il semble nécessaire de s'interroger sur le devenir des données produites dans le cas de la recherche publique, lorsque des publications sont entreposées sur de telles plateformes. Lorsqu'un chercheur est employé par une institution, le fruit de son travail doit être conservé par l'institution elle-même ${ }^{6}$, qui veille à la sécurité et à la pérennité des données.

Par ailleurs, à cette question s'ajoute celle des conditions imposées par les éditeurs scientifiques à leurs auteurs. Le dépôt immédiat sur les réseaux sociaux peut fréquemment entrer en conflit avec la politique d'une revue imposant un embargo. Elsevier n'a pas hésité par le passé à obliger Academia.edu à retirer de nombreux documents, qui n’avaient pas à y être téléchargés (21).

\footnotetext{
${ }^{6}$ En France, le Code du Patrimoine est très clair à ce sujet : les archives publiques sont les « documents qui procèdent de l'activité, dans le cadre de leur mission de service public, de l'Etat, des collectivités territoriales, des établissements publics et des autres personnes morales de droit public ou des personnes de droit privé chargées d'une telle mission ». (Article L211-4)

http://www.legifrance.gouv.fr/affichCodeArticle.do?cidTexte=LEGITEXT000006074236\&idArticle=LEGIARTI000006845562\& dateTexte=\&categorieLien $=$ cid
} 


\section{Une large méconnaissance du droit en matière de publications scientifiques}

Dans l'étude conduite par le consortium Couperin, les chercheurs étaient interrogés sur leur connaissance de la politique des données des réseaux sociaux dont ils étaient les utilisateurs. En sciences de la vie, il ressort que seulement $11 \%$ des chercheurs sont conscients du devenir de leurs données lorsqu'ils déposent des documents sur ses sites. Relevant que les « conditions [d'utilisation] ne laissent aucune place à l'utilisateur quant à la propriété de ses données mais toute latitude aux porteurs des [réseaux sociaux de la recherche] pour développer des affaires basées sur les données échangées sur leurs réseaux ", les auteurs de l'étude s'alertent de cette méconnaissance ; "ce point doit interroger la communauté scientifique alors que les chercheurs semblent très disposés à confier aux sociétés privées en charge des RSDR, un ensemble de données importantes pour leur recherche » (6).

\section{Le travail d'équipe au second plan}

La tension entre l'individu et le collectif constitue un autre biais introduit par ces sites. Sur les réseaux spécialisés, les institutions n'ont pas la possibilité de créer des pages, qui leur permettraient de fédérer l'ensemble de la communauté de recherche. De fait, si les universités et les organismes de recherche sont relativement bien signalés, les laboratoires sont pour leur part tout bonnement gommés. Cela revient, se désolait William Berthomière, géographe et directeur de recherche au CNRS à occulter le travail conduit par une équipe et à valoriser seulement un individu. William Berthomière ajoutait que ces outils prennent place dans le vide laissé par les institutions (22). Dans une étude réalisée au mois de mars, la direction de l'information scientifique et technique du CNRS a montré que les éditeurs s'engouffrent eux aussi sur ce créneau, cherchant désormais à développer des plates-formes de services, pour retenir un maximum de chercheurs (23).

\section{Visibilité ou autorité ?}

Les réseaux sociaux prêtent parfois le flanc à l'agacement. Une grande visibilité sur un réseau social ne correspond pas nécessairement à une reconnaissance dans le milieu scientifique. Neil Hall, chercheur à l'université de Liverpool, a d'ailleurs imaginé pour le prouver le K-index, du nom de 
I'héroïne de télé-réalité Kim Kardashian. Le K-index mesure l'écart entre la popularité d'un chercheur sur les réseaux sociaux et son autorité dans sa discipline. Les écarts ont parfois été grands et l'article a eu le mérite de pointer l'artificialité de l'impact de ces sites, qui relèvent de la logique de communication, bien davantage que de la rigueur scientifique (24). D’autres voix sont plus nuancées et pensent qu'il ne faudrait pas toutefois prêter plus d'importance à l'impact de ces outils qu'ils n'en ont réellement, mettant en avant le fait que les réseaux qui se mettent en place sur ces plateformes renforcent finalement les hiérarchies traditionnelles (17). II faut noter aussi qu'un profil sur un réseau social, s'il renforce la notoriété de son auteur, n'entrera nullement en compte pour son évaluation qui, elle, demeure établie à partir des outils traditionnels et, de plus en plus, conditionnée au dépôt en archive ouverte ${ }^{7}$.

\section{Accompagner l'utilisation}

L'utilisation des réseaux sociaux numériques de la recherche semble de fait devoir bénéficier d'un accompagnement. Les doctorants, notamment, constituent un public à sensibiliser à cette question qui touche à la fois à la gestion d'une identité numérique et à la question du devenir des données produites dans le cadre d'une recherche financée sur projet. Les bibliothèques universitaires sont de plus en plus nombreuses à s'emparer de la question et à proposer des formations ou des guides sur les réseaux sociaux. Les bibliothèques de l'Université Versailles Saint-Quentin, par exemple, proposent une offre de formation individualisée. Elles ont créé sur le site deux rubriques fournies, « Vos publications» (25) et "Services aux chercheurs» (26), dans lesquelles sont abordées les manières de les rendre accessibles et visibles. Sur le site Form@doct, site dédié à la formation des doctorants, I'Université européenne de Bretagne a consacré plusieurs pages aux réseaux sociaux, généralistes comme scientifiques. La question de la visibilité est abordée, de même que celles des données. A travers ces quelques pages, les enjeux de ces outils, tant leurs indéniables atouts que leurs limites, sont abordés pour que les doctorants puissent en faire une utilisation éclairée (27).

\footnotetext{
${ }^{7}$ Une archive ouverte est un entrepôt de publications scientifiques librement accessibles. L'accessibilité des documents est garantie par des normes d'interopérabilité, qui permettent l'indexation et le moissonnage du contenu du réservoir par des moteurs de recherche. Une archive ouverte peut être disciplinaire ou institutionnelle.
} 


\section{Établir des conditions d'utilisation}

Certaines institutions ont commencé à se saisir de la question des réseaux sociaux. Depuis plusieurs années, la $\mathrm{CNIL}^{8}$ émet des réserves sur les données personnelles collectées par les réseaux généralistes, mais elle n’a encore rien publié sur les réseaux spécialisés pour les chercheurs, dont les conditions générales d'utilisation mériteraient pourtant un examen approfondi. De leur côté, les universités commencent à mettre en place des chartes d'utilisation, on peut citer celle de l'université Paris Dauphine (28), des médias sociaux pour leurs étudiants, mais, là encore, elles concernent les réseaux sociaux généralistes. Enfin, certaines institutions, notamment dans la fonction publique territoriale, font signer à leurs agents des chartes d'utilisation professionnelle des médias sociaux. La préoccupation des établissements, tant en ce qui concerne les données des agents que l'image de l'institution, est réelle. Pour l'heure, les universités et les organismes de recherche n'ont pas encore émis de recommandations concernant les réseaux sociaux numériques de la recherche. Le téléchargement de la production scientifique sur ces sites n'est pourtant pas un acte anodin, dans la mesure où les chercheurs sont liés aux conditions exigées par les organismes financeurs de leurs travaux, à leur laboratoire et leur institution, à l'éditeur qui publie le fruit de leur recherche. De la même façon que les modalités de dépôt sur des archives ouvertes ont été clarifiées grâce à un site comme Sherpa/Romeo ${ }^{9}$, le téléchargement des articles sur les réseaux sociaux gagnerait, lui aussi, à bénéficier de pareil traitement.

\section{Renforcer la visibilité personnelle et institutionnelle}

L'expérience des bibliothécaires et des documentalistes aujourd'hui est souvent la même d'un établissement à l'autre. Certains chercheurs, notamment parmi les plus jeunes, ne font pas la

\footnotetext{
${ }^{8}$ La Commission nationale de l'informatique et des libertés est une autorité administrative indépendante, créée en France en 1978. Elle veille à ce que les libertés individuelles restent garanties dans le cadre des utilisations de l'informatique en France et travaille à la protection des données à caractère personnel.

${ }^{9}$ Sherpa/Romeo est une organisation, portée par une trentaine d'institutions partenaires, dont la tâche consiste à recenser les politiques des éditeurs concernant les dépôts dans une archive ouverte institutionnelle. Le site détaille pour chaque éditeur la version de l'article qui peut être déposé en archive instutionnelle, ainsi que l'éventuel embargo avant qu'il soit accessible. http://www.sherpa.ac.uk/romeo/
} 
différence entre la mise en ligne d'une publication sur un réseau social et son dépôt sur une archive ouverte. Eux se satisfont de voir que la publication est accessible, alors qu'un simple téléchargement sur un espace collaboratif privé ne peut remplacer le dépôt sécurisé sur un entrepôt institutionnel et ne résout pas la question des homonymes.

\section{Nouveaux identifiants}

Des identifiants pour les auteurs, portés par les institutions, existent depuis quelques années. Certes, ces outils pâtissent de l'absence de fonctionnalités de "réseautage ", mais ils offrent pourtant des possibilités intéressantes pour l'impact des publications.

ORCID est un système d'identifiants porté par une centaine d'institutions internationales. Il permet à chaque chercheur de créer, puis de disposer, d'une page actualisée avec ses publications. La création d'un identifiant prémunit l'auteur contre les problèmes d'homonymie, puisque le chercheur peut modifier sa page et y indiquer plusieurs « formes auteur ${ }^{10}$ si besoin. Les chercheurs peuvent ajouter leurs publications ou les récupérer sur les grandes bases de données partenaires du site. Les éditeurs sont nombreux à travailler avec ORCID, dans la mesure où ces identifiants leur permettent d'obtenir des données auteur plus fines pour le calcul du facteur d'impact. ORCID accepte le référencement des publications, mais aussi des données de recherche. Le site offre la possibilité de rechercher automatiquement les publications sur un certain nombre de bases de données, dont PubMed, pour que le chercheur n'ait plus qu'à valider les imports de ses publications (29). Le principal organisme finançant la recherche au Portugal a récemment décidé d'exiger des chercheurs qu'ils s'inscrivent sur le site. Leur référencement s'est rapidement amélioré et l'expérience s'est révélée concluante pour la visibilité des chercheurs comme pour le classement de leurs publications (30).

De son côté, HAL, la principale plateforme d'archives ouvertes en France, a mis en place l'IdHAL, un identifiant pérenne pour les chercheurs. Là encore, c'est à l'auteur que revient la démarche de créer

\footnotetext{
${ }^{10}$ Les « formes auteur " concernent les différentes manières dont un nom a pu être écrit dans une signature. Le cas le plus classique concerne le nom d'épouse, mais on trouve également des cas fréquent de prénom inscrit en entier ou réduit à l'initiale. Ces différences peuvent conduire un même auteur à être considéré comme deux personnes distinctes, ou à créer des confusions sur deux noms identiques avec les mêmes initiales de prénom. Les identifiants pérennes permettent de désambiguïser ces cas.
} 
sa page et de préciser les différentes formes auteur le concernant. Une fois la page générée, il est possible d'en faire un véritable CV scientifique, auquel sont attachés l'ensemble des publications du chercheur sur HAL. II est également possible de faire figurer sur sa page ses autres identifiants chercheur (par exemple, ORCID et ou ResearchGate) (31). L'outil est encore en développement pour permettre plus d'interopérabilité avec d'autres plateformes. Il y a là les prémices de fonctionnalités très intéressantes.

\section{Les archives ouvertes}

Lorsqu'il dépose en archives ouvertes, l'auteur est assuré que sa publication reste sous la protection du droit d'auteur de son pays. Il peut de surcroît choisir le type de licence de sa publication (Creative Commons, par exemple). En matière d'archivage, le dépôt est pérenne et sécurisé et le document est conservé au CINES. La visibilité des archives ouvertes, qui ressortent certes moins bien dans les moteurs de recherche, reste bonne : les documents sont bien référencés sur Google scholar et ils sont signalés dans de nombreux dépôts ouverts à travers le monde. Notons enfin qu'HAL fait partie des portails d'archives ouvertes les plus connus à l'international (32). A Liège, les résultats d'une enquête après la mise en place du mandat de dépôt dans l'archive ouverte de l'université ont montré que les chercheurs sont davantage cités. L'impact est réel, comme l'université l'a constaté en 2011 en comparant dans Scopus ${ }^{11}$ le nombre de citations des publications figurant dans son archive institutionnelle à celles n'y figurant pas (33). De fait, cette université conseille désormais aux chercheurs d'avoir recours aux réseaux sociaux de la recherche pour signaler leurs publications et créer des opportunités de collaboration avec d'autres chercheurs, sans pour autant y déposer le texte intégral qui est réservé à l'archive institutionnelle, comme l'expliquait Paul Thirion, directeur des bibliothèques de l'université de Liège, lors d'une récente journée d'étude consacrée à la bibliométrie (34). L'université d'Angers a elle aussi ouvert une archive institutionnelle, baptisée Okina (35), et mis en place un mandat de dépôt pour l'ensemble des publications de ses chercheurs depuis 2008. Okina permet d'effectuer des recherches tant par laboratoire que par chercheurs. Pour les

\footnotetext{
${ }^{11}$ http://www.scopus.com/
} 
laboratoires, cela permet d'avoir une page toujours actualisée qui reflète le travail produit. Les chercheurs, pour leur part, disposent d'une bibliographie complète de leurs travaux, à laquelle ils peuvent ajouter une présentation de leur parcours et un $\mathrm{CV}^{12}$. Cette solution a le mérite de mettre en valeur les chercheurs comme leurs laboratoires, en les inscrivant dans un écosystème qui fait ressortir les dynamiques de recherche et les partenariats.

L'étude conduite par le consortium Couperin a montré que les chercheurs sont parfaitement conscients de ces enjeux, notamment ce qui concerne la fiabilité des dépôts en archives ouvertes, même s'ils ne prennent pas encore systématiquement le temps d'y faire figurer leurs publications (6). Des initiatives comme celle d'Angers et de Liège, dont la valeur ajoutée est indéniable, seront peut-être de nature à impulser un mouvement plus important en faveur du dépôt en archives ouvertes.

Si le modèle traditionnel de validation de l'article n'est pas remis en cause, on remarque néanmoins l'émergence des nouveaux acteurs. Les réseaux sociaux numériques de la recherche se sont imposés dans le paysage des publications scientifiques de manière très rapide. De ce point de vue, la revue n'est plus le seul vecteur, désormais concurrencée par ces médias de l'instantanéité, plus accessibles, mais moins fiables. On note également une tendance à la transformation du modèle traditionnel qui, centré qu'il était autour d'un titre de revue ou d'un sujet, se focalise désormais sur l'individu. Ces évolutions, importantes depuis environ cinq ans, ne sont pas sans poser la question, tant de la validation des documents, qui sont lus, cités, sur ces réseaux, que de la protection des publications de la recherche publique. La Direction de l'information scientifique et technique du CNRS, dans une lettre d'information récente, a d'ailleurs invité les établissements de recherche à trancher « le nœud gordien du droit d'auteur applicable à la publication scientifique ", dans la mesure où les éditeurs

\footnotetext{
${ }^{12}$ Exemple de la page d’Eric Levillain sur Okina : http://okina.univ-angers.fr/eric.levillain
} 
eux-mêmes commencent à se préoccuper de voir tant de publications diffusées sur les réseaux sociaux spécialisés (36). Les chercheurs, pourtant, fourmillent d'idées pour développer un outil ouvert et interopérable, adapté à leurs besoins d'échanges de publications. Dans leurs commentaires au questionnaire réalisé par le consortium Couperin, ils ont dessiné l'outil idéal pour eux. International, interactif, il leur permettrait d'échanger dans un espace porté par les institutions publiques, garantissant la pérennité et la sécurité des données déposées. II s'agit peut-être d'une piste pour l'avenir (6). 


\section{Bibliographie}

1. Chantrel F, Coëffé T, Ropars F. Chiffres réseaux sociaux - 2014 [Internet]. Le blog du Modérateur. [cité 12 oct 2014]. Disponible sur: http://www.blogdumoderateur.com/chiffresreseaux-sociaux/

2. Boyd D. Social network sites: my definition [Internet]. Many 2 Many. 2006 [cité 2 avr 2015]. Disponible sur: http://many.corante.com/archives/2006/11/12/social_network_sites_my_definition.php

3. Merzeau L. La présence plutôt que l'identité. Doc - Sci Inf. 2010;47(1):32-3.

4. Merzeau L. Traces captées, traces éditorialisées [Internet]. Louise Merzeau. [cité 23 oct 2014]. Disponible sur: http://merzeau.net/traces-captees-editorialisees/

5. Arènes $C$. Modes de communication de la recherche aujourd'hui : quel rôle pour les bibliothécaires ? (Les) [Internet]. [Villeurbanne]: Ecole nationale supérieure des sciences de I'information et des bibliothèques; 2015 [cité 2 avr 2015]. Disponible sur: http://www.enssib.fr/bibliotheque-numerique/notices/65046-les-modes-de-communication-de-larecherche-aujourd-hui-quel-role-pour-les-bibliothecaires

6. Vignier S, Joly M, Okret-Manville C. Réseaux sociaux de la recherche et Open Access : perception des chercheurs. Etude exploratoire [Internet]. Couperin; 2014. Disponible sur: http://www.couperin.org/images/stories/openaire/Couperin_RSDR\%20et\%200A_Etude\%20explorat oire_2014.pdf

7. Flichy P. L'imaginaire d'Internet. Paris: La Découverte; 2001. 272 p.

8. Pierre J. L'identité numérique du chercheur : problématique, enjeux et outils [Internet]. 2014 [cité 9 nov 2014]. Disponible sur: http://fr.slideshare.net/idnum/lidentit-numrique-du-chercheurproblmatique-enjeux-et-outils

9. Bouchard A. Les réseaux sociaux : pratiques et enjeux dans la recherche et la carrière scientifique [Internet]. Urfist de Paris. [cité 2 nov 2014]. Disponible sur: http://urfist.enc.sorbonne.fr/ressources/edition-scientifique/les-reseaux-sociaux-pratiques-etenjeux-dans-la-recherche-et-la-carr

10. Berra A. Faire des humanités numériques. In: Pierre M, éditeur. Read/Write Book 2 : Une introduction aux humanités numériques [Internet]. Marseille: OpenEdition Press; 2012 [cité 24 oct 2014]. p. 25-43. Disponible sur: http://books.openedition.org/oep/238

11. Jensen P. Publier en libre accès, quelles opportunités pour les chercheurs et la diffusion des savoirs ? [Internet]. Youtube. 2014 [cité 2 avr 2015]. Disponible sur: https://www.youtube.com/watch?v=i_4pXWs9GjI\&feature=youtube_gdata_player

12. Deville S. Twitter et les chercheurs, l'exception française ? [Internet]. Le Monde.fr. [cité 22 juill 2014]. Disponible sur: http://www.lemonde.fr/sciences/article/2014/02/05/twitter-et-leschercheurs-I-exception-francaise_4360491_1650684.html 
13. Saint-Martin A, Lamy J. Devenir carnetiers ? Premier retour d'expérience [Internet]. Devenir historien-ne. 2014 [cité 22 mai 2014]. Disponible sur: http://devhist.hypotheses.org/2514

14. Pouyllau S. Le libre accès privatisé ? [Internet]. sp.Blog. [cité 10 avr 2015]. Disponible sur: http://blog.stephanepouyllau.org/709

15. MalariaWorld | The world's scientific and social network for malaria professionals [Internet]. [cité 2 avr 2015]. Disponible sur: http://www.malariaworld.org/

16. Bester E. Les médias sociaux dans la médiation grand public et pour la communication scientifique entre pairs : de " l'information overload » au " tools overload » [Internet].

Academia.edu. [cité 12 oct 2014]. Disponible sur:

http://www.academia.edu/4473343/Bester_E._Les_medias_sociaux_dans_la_mediation_grand_publ ic_et_pour_la_communication_scientifique_entre_pairs_de_linformation_overload_au_tools_overlo ad_

17. Jordan K. Academics and their online networks: Exploring the role of academic social networking sites. First Monday [Internet]. 25 oct 2014 [cité 10 mars 2015];19(11). Disponible sur: http://firstmonday.org/ojs/index.php/fm/article/view/4937

18. Thelwall M, Kousha K. ResearchGate: Disseminating, communicating, and measuring Scholarship? J Assoc Inf Sci Technol. 1 juin 2014;n/a - n/a.

19. Terms of use [Internet]. Academia.edu. [cité 2 avr 2015]. Disponible sur: http://www.academia.edu/terms

20. Bianchini L. Rachat de l'assistant de gestion bibliographique et réseau social Mendeley par Elsevier [Internet]. Agence Science-Presse. [cité 10 avr 2015]. Disponible sur: http://www.sciencepresse.qc.ca/blogue/2013/04/15/rachat-lassistant-gestion-bibliographiquereseau-social-mendeley-elsevier

21. Campbell L. Elsevier in open access row [Internet]. The bookseller. 2013 [cité 2 avr 2015]. Disponible sur: http://www.thebookseller.com/news/elsevier-open-access-row

22. Berthomière $W$. Regards croisés sur la journée : le point de vue d'un chercheur impliqué, d'un évaluateur embarrassé ! [Internet]. UPtv. [cité 12 déc 2014]. Disponible sur: http://uptv.univpoitiers.fr/program/reseaux-sociaux-de-chercheursetnbsp-quelle-

visibiliteetnbsp/video/4415/regards-croises-sur-la-journee-le-point-de-vue-d-un-chercheur-impliqued-un-evaluateur-embarrasse/index.html

23. DIST, CNRS. L'Edition de sciences à l'heure numérique : dynamiques en cours [Internet]. Disponible sur: http://www.cnrs.fr/dist/z-outils/documents/Distinfo2/Distetude2.pdf

24. Hall N. The Kardashian index: a measure of discrepant social media profile for scientists. Genome Biol. 30 juill 2014;15(7):424.

25. Vos publications [Internet]. Bibliothèques universitaires - Université Versailles Saint-Quentin. [cité 9 nov 2014]. Disponible sur: http://www.bib.uvsq.fr/vos-publications\#identite

26. Services aux chercheurs [Internet]. Bibliothèques universitaires - Université Versailles Saint- 
Quentin. [cité 9 nov 2014]. Disponible sur: http://www.bib.uvsq.fr/services-aux-chercheurs

27. Form@doct [Internet]. Université européenne de Bretagne. [cité 9 nov 2014]. Disponible sur: http://guides-formadoct.ueb.eu/

28. Université Paris Dauphine. Charte d'utilisation des médias sociaux [Internet]. Disponible sur: http://www.dauphine.fr/fileadmin/mediatheque/Communication/charte/Charte_d_utilisation_des_ medias_sociaux_UPD.pdf

29. ORCID | Connecting Research and Researchers [Internet]. [cité 13 oct 2014]. Disponible sur: http://orcid.org/

30. Blanchard A. Pour une meilleure visibilité de la recherche française $3 / 3:$ Annuaires de chercheurs et valorisation de l'expertise des laboratoires [Internet]. Deuxième labo. [cité 2 avr 2015]. Disponible sur: http://www.deuxieme-labo.fr/article/pour-une-meilleure-visibilite-de-la-recherchefrancaise-33-annuaires-de-chercheurs-et-valorisation-de-lexpertise-des-laboratoires/

31. L'IdHAL, pour quoi faire et comment s'y prendre [Internet]. Le blog du CCSD. [cité 2 avr 2015]. Disponible sur: http://blog.ccsd.cnrs.fr/2015/03/idhal-pour-quoi-faire-et-comment-syprendre/

32. Top Institutionals [Internet]. Ranking Web of Repositories. [cité 2 avr 2015]. Disponible sur: http://repositories.webometrics.info/en/top_Inst

33. Thirion P. ORBi : pari tenu ? 2012 [cité 2 avr 2015]. Disponible sur: http://orbi.ulg.ac.be/handle/2268/132713

34. Journée d'information : «Bibliométrie, scientométrie et métriques alternatives : quels outils pour quelles stratégies? " [Internet]. ADBU - Association des directeurs et des personnels de direction des bibliothèques universitaires. 2015 [cité 3 avr 2015]. Disponible sur:

https://adbu.fr/journee-dinformation-bibliometrie-scientometrie-et-metriques-alternatives-quelsoutils-pour-quelles-strategies/

35. Okina : Open Knowledge, INformation, Access [Internet]. Université d'Angers. [cité $10 \mathrm{avr}$ 2015]. Disponible sur: http://okina.univ-angers.fr/

36. DIST, CNRS. L'association internationale des éditeurs scientifiques lance une consultation sur les logiques de partage des articles scientifiques entre chercheurs [Internet]. 2015. Disponible sur: http://www.cnrs.fr/dist/z-outils/documents/Distinfo2/Distinf10.pdf 